\title{
B-Spline Approximation of Ship Waves on the Free Surface
}

\author{
Fang Li, Hui Li, Huilong Ren \\ College of Shipbuilding Engineering, Harbin Engineering University, Harbin, China \\ Email: lifang199011@163.com
}

Received December 2014

\begin{abstract}
We consider the problem of a ship advancing in waves. In this method, the zone of free surface in the vicinity of body is discretized. On the discretized surface, the first-order and second-order derivatives of ship waves are represented by the B-Spline formulae. Different ship waves are approximated by cubic B-spline and the first and second order derivates of incident waves are calculated and compared with analytical value. It approves that this numerical method has sufficient accuracy and can be also applied to approximate the velocity potential on the free surface.
\end{abstract}

\section{Keywords}

Cubic B-Spline, Free Surface Grid Division, Approximation of Incident Wave

\section{Introduction}

In the method for hydrodynamic analysis of floating bodies with forward speed, due to the complex boundary condition on the free surface, the integral equation involves not only the unknown velocity potential but also its first-order and second-order derivatives on the free surface. Unlike classical methods in which a waterline integral is present by using Stokes' theorem, we apply directly the free-surface condition so that the troublesome waterline integral is avoided. The first- and second-order derivatives of the velocity potential in this new method are then approximated using B-Spline method.

In this paper, the incident wave on free surface is approximated by cubic B-spline and the relationship between incident wave and its first-order and second-order partial derivatives are derived and compared with the analytical value.

This method is approved to have sufficient accuracy and can be also applied to approximate the velocity potential on the free surface.

\section{Fundamental Theory of B-Spline}

\subsection{Definition of B-Spline Curves and Surfaces}

Let the knot vector $\bar{U}$ be a set of $m+1$ non-decreasing numbers, $u_{0}<=u_{2}<=u_{3}<=\ldots<=u_{m}$. We define the $k$ th degree B-spline curve as Equation (1) [1]: 


$$
\vec{p}(u)=\sum_{i=0}^{n} \vec{d}_{i} N_{i, k}(u)
$$

where $\vec{d}_{i}$ is control vertices, $n+1$ is the number of control vertices, $N_{i, k}(u)$ is the kth degree B-spline basis functions.

The $i$ th B-spline basis function of degree $k$, written as $N_{i, k}(u)$, is defined by Cox-de Boor recursion formula as Equation (2) [2]

$$
\left\{\begin{array}{l}
N_{i, 0}(u)=\left\{\begin{array}{l}
1, u_{i} \leq u<u_{i+1} \\
0, \text { others }
\end{array}\right. \\
N_{i, k}(u)=\frac{u-u_{i}}{u_{i+k}-u_{i}} N_{i, k-1}(u)+\frac{u_{i+k+1}-u}{u_{i+k+1}-u_{i+1}} N_{i+1, k-1}(u)
\end{array}\right.
$$

In the same way, we define the B-spline surface by B-spline tensor product expansion as Equation (3) [3]

$$
\vec{p}(u, v)=\sum_{i=0}^{m} \sum_{j=0}^{n} \vec{d}_{i, j} N_{i, k}(u) N_{j, l}(v)
$$

where $N_{i, k}(u)$ is the $k$ th degree B-spline basis functions, $N_{j, l}(v)$ is the lth degree B-spline basis functions, $\vec{d}_{i, j}$ is control vertices, $m+1$ and $n+1$ are the number of control vertices in $u$ and $v$ parametric directions respectively, $u$ and $v$ are two independent parameters which monotonically increase along the respective parametric spaces.

\subsection{The de Boor Algorithm}

Given a clamped B-spline curve of degree $k$ defined by $n+1$ control points $\vec{d}_{0}, \vec{d}_{1}, \vec{d}_{2}, \ldots, \vec{d}_{n}$ and $m+1$ knots $u_{0}=u_{1}=\ldots=u_{k}=0, u_{k+1}, u_{k+2}, \ldots, u_{m-k-1}, u_{m-k}=u_{m-k+1}=\ldots=u_{m}=1$, we can compute the point on the B-spline curve by the de Boor algorithm as Equation (4) [2]

$$
\vec{p}(u)=\sum_{j=0}^{n} \vec{d}_{j} N_{j, k}(u)=\sum_{j=i-k}^{i-l} \vec{d}_{j}^{l} N_{j, k-l}(u)=\cdots=d_{i-k}^{k} \quad u \in\left[u_{i}, u_{i+1}\right] \in\left[u_{k}, u_{n+1}\right]
$$

where

$$
\bar{d}_{j}^{l}=\left\{\begin{array}{l}
\bar{d}_{j}, l=0 \\
\left(1-\alpha_{j}^{l}\right) \bar{d}_{j}^{l-1}+\alpha_{j}^{l} \bar{d}_{j+1}^{l-1}, j=i-k, i-k+1, \cdots, i-l ; l=1,2, \cdots, k
\end{array} \quad \alpha_{j}^{l}=\frac{u-u_{j+1}}{u_{j+k+1}-u_{j+l}}\right.
$$

\subsection{Derivatives of B-Spline Curves and Surfaces}

Given a clamped B-spline curve of degree $k$, we can compute $r$ order derivatives $\vec{p}^{(r)}(u)$ as Equation (6) [2]:

$$
\vec{p}^{(r)}(u)=\frac{d^{r}}{d u^{r}} \sum_{j=0}^{n} \vec{d}_{j} N_{j, k}(u)=\sum_{j=i-k}^{i-r} \vec{d}_{j}^{r} N_{j, k-r}(u) \quad u \in\left[u_{i}, u_{i+1}\right] \in\left[u_{k}, u_{n+1}\right]
$$

where

$$
\bar{d}_{j}^{l}=\left\{\begin{array}{l}
\bar{d}_{j}, l=0 \\
(k-l+1) \frac{\bar{d}_{j+1}^{l-1}-\bar{d}_{j}^{l-1}}{u_{j+k+1}-u_{j+l}}, j=i-k, i-k+1, \cdots, i-r ; l=1,2, \cdots, r
\end{array}\right.
$$

\subsection{B-Spline Curves and Surfaces Approximation}

B-spline curve approximation can be stated as the problem of constructing a B-spline curve passing through a set of fixed points $\bar{p}_{i}(i=0, \ldots, m)$. Let us take cubic B-spline as an example, When the points $\bar{p}_{i}(i=0, \ldots, m)$ and a knot vector $\vec{U}$ of a B-spline curve are given, the problem is solving a set of linear equations as Equation (8) [4]: 


$$
\vec{p}\left(u_{i}\right)=\sum_{j=0}^{n} \vec{d}_{j} N_{j, 3}\left(u_{i}\right)=\sum_{j=i-3}^{i} \vec{d}_{j} N_{j, 3}\left(u_{i}\right)=\vec{p}_{i-3}, \quad i=3,4, \ldots, n
$$

where $\vec{d}_{j}(j=0, \ldots, n, n=m+2)$ are unknown control points.

\section{The Details of Study Method}

\subsection{Approximation of Incident Wave on the Free Surface}

We choose a set of points $\vec{p}_{i, j}=(x, y, z) \quad(i=0, \ldots, 10, j=0, \ldots, 41)$ on the free surface. $(x, y)$ is position on the surface, $z$ is the height of incident wave and it can be defined as Equation (9)

$$
z=A \cos [k(x \cos \beta-y \sin \beta)+\omega t+\varepsilon]
$$

where $A$ is the amplitude of the incident wave, $k$ is wave number, $\beta$ is wave heading, $t$ is time, $\omega$ is circle frequency and $\varepsilon$ is phase angle.

Let $\vec{p}_{i}(i=0, \ldots, n)$ denote the $i$ th contour whose points are given as $\vec{p}_{i, j} \quad(j=0, \ldots, m)$. Each contour is considered to be open and the number of contour points is equal.

When the incident wave is given as $z=4 \cos (0.0785 x)$, the distribution of points $\vec{p}_{i, j} \quad(i=0, \ldots, 10, j=$ $0, \ldots, 41)$ on the free surface is shown as Figure 1.

We choose cubic B-spline to approximate the free surface. The free surface is divided into two patches, then each patch is described by cubic B-spline tensor product expansion as Equation (10). Figure 2 shows the wave surface approximated by cubic B-spline

$$
\vec{p}(u, v)=\sum_{i=0}^{m} \sum_{j=0}^{n} \vec{d}_{i, j} N_{i, 3}(u) N_{j, 3}(v), 0 \leq u, v \leq 1
$$

\subsection{Calculation of the Derivatives of Incident Wave Based on B-Spline}

The points on the surface can be described as Equation (11):

$$
x=x(u, v) ; y=y(u, v) ; z=z(u, v)
$$

The first and second order partial derivatives of incident wave $\frac{\partial z}{\partial u}$ and $\frac{\partial^{2} z}{\partial^{2} u}$ can be written as Equations (12) and (13)

$$
\begin{gathered}
\frac{\partial z}{\partial u}=\frac{\partial z}{\partial x} \cdot \frac{\partial x}{\partial u} \\
\frac{\partial^{2} z}{\partial^{2} u}=\frac{\partial^{2} z}{\partial^{2} x} \cdot\left(\frac{\partial x}{\partial u}\right)^{2}+\frac{\partial z}{\partial x} \cdot \frac{\partial^{2} x}{\partial^{2} u}
\end{gathered}
$$

We can calculate the value of $\frac{\partial x}{\partial u}, \frac{\partial z}{\partial u}, \frac{\partial^{2} x}{\partial^{2} u}, \frac{\partial^{2} z}{\partial^{2} u}$ by the de Boor algorithm which is introduced in Sec-

Figure 1. The distribution of points on the free surface.

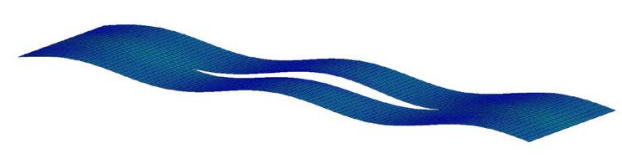

Figure 2. The wave surface approximated by cubic B-spline. 
tion 2.2 and 2.3. Then the value of and $\frac{\partial^{2} z}{\partial^{2} x}$ are obtained through Equation (13). Table 1 shows the value of $\frac{\partial z}{\partial x}$ and $\frac{\partial^{2} z}{\partial^{2} x}$ compare with analytical value at $v=0.5 . E_{r}$ is defined as Equation (14):

$$
E_{r}=\left|\frac{f_{\text {num }}-f_{\text {analytical }}}{\max \left\{f_{\text {num }}\right\}}\right|
$$

\subsection{Approximation of Different Incident Wave on The free Surface}

We choose the same points $\vec{p}_{i, j}=(x, y, z) \quad(i=0, \ldots, 10, j=0, \ldots, 41)$ which is mentioned in Section 3.1 to describe the free surface. When the wave heading $\beta$ is $45^{\circ}$, we define the incident wave as Equation (15)

$$
z=4 \cos [0.0785(x \cos 45-y \sin 45)]
$$

Then we use the cubic B-spline to approximate the free surface as we do in Section 3.1. Figure 3 show the wave surface approximated by cubic B-spline.

Then we can calculate the value of $\frac{\partial z}{\partial x}$ and $\frac{\partial z}{\partial y}$ through Equation (16)

$$
\left[\begin{array}{l}
\frac{\partial z}{\partial x} \\
\frac{\partial z}{\partial y}
\end{array}\right]=\left[\begin{array}{ll}
\frac{\partial x}{\partial u} & \frac{\partial y}{\partial u} \\
\frac{\partial x}{\partial v} & \frac{\partial y}{\partial v}
\end{array}\right]^{-1}\left[\begin{array}{l}
\frac{\partial z}{\partial u} \\
\frac{\partial z}{\partial v}
\end{array}\right]
$$

In the same way, the value of $\frac{\partial^{2} z}{\partial x^{2}}$ and $\frac{\partial^{2} z}{\partial y^{2}}$ can be calculated through Equation (16), Table 2 shows the

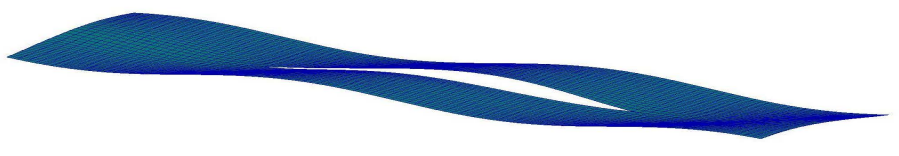

Figure 3. The wave surface approximated by cubic B-spline at $\beta=45^{\circ}$.

Table 1. Computed values and comparison to the analytical value at $v=0.5$.

\begin{tabular}{ccccccc}
\hline \multirow{2}{*}{$u$} & \multicolumn{5}{c}{$v=0.5$} \\
\cline { 2 - 7 } & $\left(Z_{x}\right)_{\text {uum }}$ & $\left(Z_{x}\right)_{\text {analyical }}$ & $E_{r}$ & $\left(Z_{x}\right)_{\text {mum }}$ & $\left(Z_{x}\right)_{\text {analyical }}$ & $E_{r}$ \\
\hline 0 & $3.1385 \mathrm{E}-01$ & $3.1400 \mathrm{E}-01$ & $4.7431 \mathrm{E}-04$ & $7.6716 \mathrm{E}-06$ & $-9.8143 \mathrm{E}-05$ & $4.2804 \mathrm{E}-03$ \\
0.1 & $-4.8933 \mathrm{E}-02$ & $-4.8813 \mathrm{E}-02$ & $3.8206 \mathrm{E}-04$ & $-2.4175 \mathrm{E}-02$ & $-2.4349 \mathrm{E}-02$ & $7.0685 \mathrm{E}-03$ \\
0.2 & $-3.1167 \mathrm{E}-01$ & $-3.1164 \mathrm{E}-01$ & $7.3223 \mathrm{E}-05$ & $3.0150 \mathrm{E}-03$ & $3.0139 \mathrm{E}-03$ & $4.3419 \mathrm{E}-05$ \\
0.3 & $2.5385 \mathrm{E}-02$ & $2.5265 \mathrm{E}-02$ & $3.8278 \mathrm{E}-04$ & $2.4606 \mathrm{E}-02$ & $2.4569 \mathrm{E}-02$ & $1.5064 \mathrm{E}-03$ \\
0.4 & $3.1375 \mathrm{E}-01$ & $3.1376 \mathrm{E}-01$ & $3.9942 \mathrm{E}-06$ & $-9.7945 \mathrm{E}-04$ & $-9.7195 \mathrm{E}-04$ & $3.0321 \mathrm{E}-04$ \\
0.5 & $-3.3066 \mathrm{E}-03$ & $-3.2561 \mathrm{E}-03$ & $1.6085 \mathrm{E}-04$ & $-2.5005 \mathrm{E}-02$ & $-2.4648 \mathrm{E}-02$ & $1.4440 \mathrm{E}-02$ \\
0.6 & $-3.1394 \mathrm{E}-01$ & $-3.1394 \mathrm{E}-01$ & $2.2539 \mathrm{E}-06$ & $-4.8887 \mathrm{E}-04$ & $-4.7954 \mathrm{E}-04$ & $3.7742 \mathrm{E}-04$ \\
0.7 & $-1.8954 \mathrm{E}-02$ & $-1.8852 \mathrm{E}-02$ & $3.2335 \mathrm{E}-04$ & $2.4721 \mathrm{E}-02$ & $2.4605 \mathrm{E}-02$ & $4.6962 \mathrm{E}-03$ \\
0.8 & $3.1236 \mathrm{E}-01$ & $3.1235 \mathrm{E}-01$ & $2.4578 \mathrm{E}-05$ & $2.5142 \mathrm{E}-03$ & $2.5244 \mathrm{E}-03$ & $4.1486 \mathrm{E}-04$ \\
0.9 & $4.2845 \mathrm{E}-02$ & $4.2365 \mathrm{E}-02$ & $1.5289 \mathrm{E}-03$ & $-2.5056 \mathrm{E}-02$ & $-2.4424 \mathrm{E}-02$ & $2.5599 \mathrm{E}-02$ \\
1 & $-3.1388 \mathrm{E}-01$ & $-3.1400 \mathrm{E}-01$ & $3.7208 \mathrm{E}-04$ & $-4.5533 \mathrm{E}-06$ & $-9.8143 \mathrm{E}-05$ & $3.7858 \mathrm{E}-03$ \\
\hline
\end{tabular}


Table 2. Computed values and comparison to the analytical value at $u=0.1$.

\begin{tabular}{ccccccc}
\hline & \multicolumn{5}{c}{$u=0.1$} \\
\cline { 2 - 7 } & $\left(Z_{x}\right)_{\text {num }}$ & $\left(Z_{x}\right)_{\text {analyical }}$ & $E_{r}$ & $\left(Z_{x}\right)_{\text {num }}$ & $\left(Z_{x}\right)_{\text {analyical }}$ & $E_{r}$ \\
\hline 0 & $-2.0700 \mathrm{E}-01$ & $-2.0704 \mathrm{E}-01$ & $1.8920 \mathrm{E}-01$ & $4.5000 \mathrm{E}-03$ & $4.4454 \mathrm{E}-03$ & $1.2133 \mathrm{E}-02$ \\
0.1 & $-1.8170 \mathrm{E}-01$ & $-1.8552 \mathrm{E}-01$ & $1.8520 \mathrm{E}-01$ & $6.7000 \mathrm{E}-03$ & $6.7666 \mathrm{E}-03$ & $9.9432 \mathrm{E}-03$ \\
0.2 & $-1.5930 \mathrm{E}-01$ & $-1.6145 \mathrm{E}-01$ & $1.7000 \mathrm{E}-01$ & $8.4000 \mathrm{E}-03$ & $8.4564 \mathrm{E}-03$ & $6.7086 \mathrm{E}-03$ \\
0.3 & $-1.3700 \mathrm{E}-01$ & $-1.3712 \mathrm{E}-01$ & $1.4140 \mathrm{E}-01$ & $9.7000 \mathrm{E}-03$ & $9.6894 \mathrm{E}-03$ & $1.0950 \mathrm{E}-03$ \\
0.4 & $-1.1370 \mathrm{E}-01$ & $-1.1319 \mathrm{E}-01$ & $1.1110 \mathrm{E}-01$ & $1.0600 \mathrm{E}-02$ & $1.0599 \mathrm{E}-02$ & $9.3605 \mathrm{E}-05$ \\
0.5 & $-8.9700 \mathrm{E}-02$ & $-8.9578 \mathrm{E}-02$ & $8.6100 \mathrm{E}-02$ & $1.1200 \mathrm{E}-02$ & $1.1273 \mathrm{E}-02$ & $6.5371 \mathrm{E}-03$ \\
0.6 & $-6.5400 \mathrm{E}-02$ & $-6.5674 \mathrm{E}-02$ & $6.8200 \mathrm{E}-02$ & $1.1700 \mathrm{E}-02$ & $1.1769 \mathrm{E}-02$ & $5.9234 \mathrm{E}-03$ \\
0.7 & $-4.0800 \mathrm{E}-02$ & $-4.1040 \mathrm{E}-02$ & $4.3700 \mathrm{E}-02$ & $1.2100 \mathrm{E}-02$ & $1.2108 \mathrm{E}-02$ & $6.9538 \mathrm{E}-04$ \\
0.8 & $-1.5600 \mathrm{E}-02$ & $-1.5557 \mathrm{E}-02$ & $1.2100 \mathrm{E}-02$ & $1.2300 \mathrm{E}-02$ & $1.2290 \mathrm{E}-02$ & $7.7338 \mathrm{E}-04$ \\
0.9 & $1.1000 \mathrm{E}-02$ & $1.1159 \mathrm{E}-02$ & $-1.5700 \mathrm{E}-02$ & $1.2200 \mathrm{E}-02$ & $1.2305 \mathrm{E}-02$ & $8.6232 \mathrm{E}-03$ \\
1 & $4.1000 \mathrm{E}-02$ & $4.1014 \mathrm{E}-02$ & $-2.4200 \mathrm{E}-02$ & $1.2100 \mathrm{E}-02$ & $1.2109 \mathrm{E}-02$ & $7.1767 \mathrm{E}-04$ \\
\hline
\end{tabular}

value of $\frac{\partial z}{\partial x}$ and $\frac{\partial^{2} z}{\partial^{2} x}$ compare with analytical value at $u=0.1$

$$
\begin{aligned}
& \frac{\partial^{2} z}{\partial u^{2}}=\frac{\partial^{2} z}{\partial x^{2}}\left(\frac{\partial x}{\partial u}\right)^{2}+\frac{\partial z}{\partial x} \frac{\partial^{2} x}{\partial u^{2}}+\frac{\partial^{2} z}{\partial y^{2}}\left(\frac{\partial y}{\partial u}\right)^{2}+\frac{\partial z}{\partial y} \frac{\partial^{2} y}{\partial u^{2}} \\
& \frac{\partial^{2} z}{\partial v^{2}}=\frac{\partial^{2} z}{\partial v^{2}}\left(\frac{\partial x}{\partial v}\right)^{2}+\frac{\partial z}{\partial x} \frac{\partial^{2} x}{\partial v^{2}}+\frac{\partial^{2} z}{\partial y^{2}}\left(\frac{\partial y}{\partial v}\right)^{2}+\frac{\partial z}{\partial y} \frac{\partial^{2} y}{\partial v^{2}}
\end{aligned}
$$

\section{Conclusion}

In this paper, we adopt cubic B-spline approximating the free surface in different incident waves, calculate the first and second order derivatives of incident wave based on B-spline theory and compare the numerical value to the analytical value. This method is approved to have sufficient accuracy but it depends on the selection of points. In addition, it also can be applied to approximate the velocity potential on the free surface.

\section{Acknowledgements}

This paper is funded by the International Exchange Program of Harbin Engineering University for Innovationoriented Talents Cultivation and MOST 2011 CB013703 plan.

\section{References}

[1] Gordon, W.J. and Riesenfeld, R.F. B-Spline Curves and Surfaces [A].

[2] De Boor, C. (1972) On Calculation with B-Splines. J. Approx. Theory.

[3] Hoschek, J. and Lasser, D. (1993) Fundamentals of Computer Aided Geometric Design. A K Peters.

[4] Farin, G. (1993) Curves and Surfaces for Computer Aided Geometric Design. Academic Press. 